\title{
Cohesive properties of crystalline solids by the generalized gradient approximation
}

\author{
X. J. Kong, ${ }^{*}$ C. T. Chan, K. M. Ho, and Y. Y. Ye ${ }^{\dagger}$ \\ Ames Laboratory-U.S. Department of Energy and Department of Physics, Iowa State University, Ames, Iowa 50011 \\ (Received 12 February 1990; revised manuscript received 2 July 1990)
}

\begin{abstract}
The cohesive properties of $\mathrm{Al}, \mathrm{C}$, and $\mathrm{Si}$ are calculated using the generalized gradient approximation (GGA) of Perdew and co-workers. Results of numerical tests of atomic total energies and ionization energies are also presented. Cohesive energies calculated with the GGA agree much better with experimental values than results calculated with the local-density approximation, which usually overbinds. The improvement is mainly due to the better error-cancellation property of GGA.
\end{abstract}

\section{INTRODUCTION}

Hohenberg and Kohn ${ }^{1}$ showed that the ground-state properties of a system of interacting electrons are determined by the charge density. Since the exact density functional for the total energy is not known, calculations are usually carried out with the local-density approximation (LDA), ${ }^{2,3}$ which approximates the exchangecorrelation energy of the electrons by

$$
E_{\mathrm{xc}}=\int d \mathbf{r} n(\mathbf{r}) \varepsilon_{\mathrm{xc}}(n(\mathbf{r}))
$$

where $n(\mathbf{r})$ is the electron density at point $\mathbf{r}$, and $\varepsilon_{\mathrm{xc}}(n)$ is the exchange-correlation energy per particle of a homogeneous electron gas with charge density $n$. There are quite a few approximations proposed for $\varepsilon_{\mathrm{xc}}(n),{ }^{4-7}$ which all give more or less the same results. Computationally, solving the LDA self-consistent equations is not more complicated than solving the Hartree equation. When it is originally formulated, the LDA is intended for systems of slowly varying charge density, but in practice, many calculations in the past decade have demonstrated that the LDA is good for ground-state properties of realistic systems, giving quite decent results and even predictions for atomic, molecular, and crystalline systems. ${ }^{8}$ It is particularly good for structural properties, prediction of ground-state crystal structures, lattice constants, bulk moduli, phonon frequencies, surface relaxation, and reconstruction. There are, however, a few welldocumented systematic errors within the local-density approximation. The one that we are most concerned with is the systematic overestimate of binding energies in molecules and crystals. There are attempts to improve LDA by adding in the lowest-order (second-order) corrections in the electron density gradient to the LDA exchange-correlation functional. This approach, commonly called the gradient expansion approximation (GEA), ${ }^{9}$ has not been successful in application to realistic problems. It was then realized ${ }^{10}$ that the $\mathrm{LDA}$, which is the zeroth-order term in the gradient series, satisfies the important sum rules that the "exchange hole" is everywhere negative and integrates to (minus) one electron. However, the GEA violates the sum rule. Langreth and $\mathrm{Mehl}^{11}$ (LM) analyzed the exchange-correlation energy in Fourier space and proposed a non-local-density function- al for exchange-correlation in the form of a gradient expansion, later extended to spin-polarized systems by $\mathrm{Hu}$ and Langreth. ${ }^{12}$ Perdew $^{13}$ analyzed the exchange hole of the GEA in real space and imposed a real-space cutoff so that the exchange-hole sum rules are satisfied. The resulting exchange functional was then cast in simple analytic form by Perdew and Wang. ${ }^{14}$ Perdew $^{15}$ also improved the correlation functional of LM by choosing a different separation of exchange and correlation (so that they go to GEA results in the slowly varying limit) and beyond-random-phase-approximation effects are also included. Following Perdew and co-workers, we will call their formulation of exchange and correlation the "generalized gradient approximation" (GGA). The LM and GGA functionals have been applied with considerable success to total energies of atoms ${ }^{16}$ and the dissociation energies of a number of diatomic molecules. ${ }^{17}$ The LM functional has also been applied to study the cohesive properties of Si (Ref. 18) and Be, ${ }^{19}$ and the cohesive energies agree much better with experiments than LDA results. Very recently, cohesive properties of a few transition elements were also considered. ${ }^{20}$ The main purpose of the present paper is to apply the GGA, which seems to have received slightly less attention than $\mathbf{L M}$ in realistic computations, to study the cohesive properties of a few elements in their crystalline state. We will not duplicate in this paper the formulas for the exchange-correlation energy and potential functional in the GGA. Interested readers may refer to Refs. 14 and 15 for the formulas. We will show that the GGA gives much better cohesive energies of the elements considered than LDA. The remaining part of the paper will be organized as follows. Numerical tests on atomic systems will be given in Sec. II. Cohesive properties of a few elements will be described in Sec. III. Section IV is the discussion.

\section{ATOMIC CALCULATIONS}

The calculations for isolated atoms are done with a Herman-Skillman-type ${ }^{21}$ code (assuming spherical symmetry and nonrelativistic). The LDA results are calculated, unless otherwise noted, using the Ceperley-Alder local exchange-correlation potential as parametrized by Perdew and Zunger. ${ }^{7}$ As a test, we have calculated the exchange and correlation energy of some atoms and ions 
TABLE I. (a) Exchange energies (in hartrees) of neutral atoms. Reference 14 uses nonrelativistic Hartree-Fock densities and the present work uses LDA charge densities. The "exact" values are quoted from Ref. 14. (b) Correlation energies of atoms and ions (in hartrees). Reference 15 uses Hartree-Fock densities and the present work uses LDA charge densities. The experimental values are quoted from Ref. 15 .

\begin{tabular}{lccc}
\hline \hline & & (a) & \\
Atom & Ref. 14 & Present & Exact \\
\hline $\mathrm{H}$ & -0.311 & -0.299 & -0.3125 \\
$\mathrm{He}$ & -1.033 & -1.009 & -1.026 \\
$\mathrm{Li}$ & -1.789 & -1.763 & -1.781 \\
$\mathrm{Be}$ & -2.68 & -2.66 & -2.67 \\
$\mathrm{Ne}$ & -12.22 & -12.15 & -12.11 \\
$\mathrm{~A}$ & -30.29 & -30.23 & -30.18 \\
$\mathrm{Zn}$ & -69.93 & -69.78 & -69.7 \\
$\mathrm{Kr}$ & -93.8 & -93.75 & -93.9 \\
$\mathrm{Xe}$ & -178.6 & -178.5 & -179.1
\end{tabular}

(b)

\begin{tabular}{lrrc} 
Atom & Ref. 15 & Present & Experiment \\
\hline $\mathrm{H}$ & -0.003 & -0.003 & 0 \\
$\mathrm{He}^{+}$ & 0.002 & 0.002 & 0 \\
$\mathrm{Li}^{2+}$ & 0.004 & 0.004 & 0 \\
$\mathrm{He}$ & -0.044 & -0.043 & -0.042 \\
$\mathrm{Li}^{+}$ & -0.045 & -0.045 & -0.044 \\
$\mathrm{Be}^{2+}$ & -0.049 & -0.048 & -0.044 \\
$\mathrm{Be}$ & -0.094 & -0.093 & -0.094 \\
$\mathrm{Ne}$ & -0.136 & -0.135 & -0.18 \\
$\mathrm{Ne}$ & -0.39 & -0.38 & -0.39 \\
$\mathrm{Ar}$ & -0.80 & -0.80 & -0.79 \\
$\mathrm{Kr}$ & -2.01 & -2.00 & 0 \\
$\mathrm{Xe}$ & -3.31 & -3.31 & 0 \\
\hline \hline
\end{tabular}

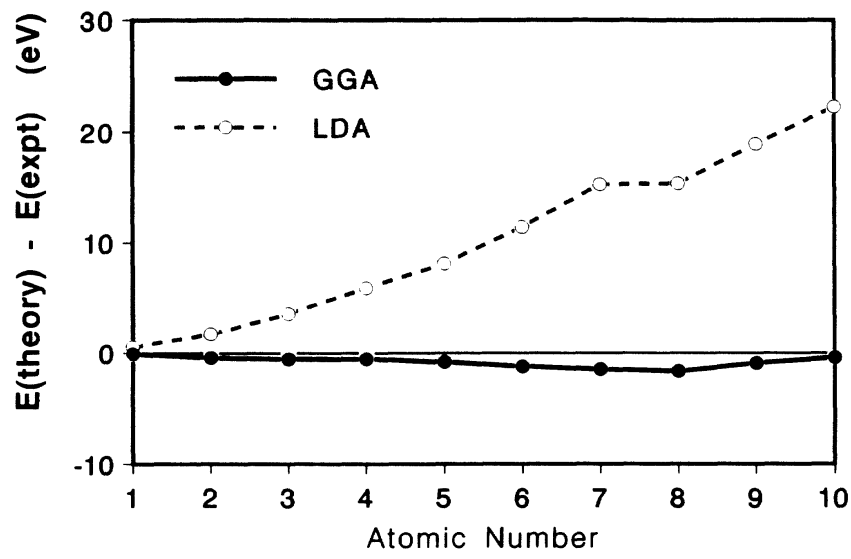

FIG. 1. Differences between theoretical and experimental [ $E$ (theory) $-E$ (expt)] atomic total energies calculated using the LDA (open circles) and the GGA (solid circles). Experimental data are from Ref. 35 . The lines serve as guides to the eye.

and compare with the corresponding values quoted in Refs. 14 and 15. Results are shown in Table I. The results of Perdew and Wang and of Perdew are basically reproduced. The small discrepancy is due to the fact that we have used the self-consistent LDA charge density to evaluate the GGA exchange and correlation functional, whereas Perdew and Wang and Perdew used HartreeFock densities. The atomic total energies are calculated for elements up to $\mathrm{Ca}$. We have restricted ourselves to lighter elements, where relativistic effects should be small and hence appropriate as test cases for GGA which is a nonrelativistic formulation. Results are listed in Table II. The calculated results are compared with "experimental" values (sum of ionization energies) in Fig. 1. We first

TABLE II. Atomic total energies (in Ry) as calculated by the LDA (with Ceperley-Alder correlation) and GGA. The GGA (NSC) values are calculated by putting the LDA charges in the GGA exchange-correlation functional.

\begin{tabular}{llrrr}
\hline \hline Element & Configuration & LDA & GGA (NSC) & GGA \\
\hline $\mathrm{H}$ & $1 s^{1}$ & 0.958 & 0.963 & 1.004 \\
$\mathrm{He}$ & $1 s^{2}$ & 5.669 & 5.827 & 5.829 \\
$\mathrm{Li}$ & $1 s^{2} 2 s^{1}$ & 14.685 & 14.988 & 14.991 \\
$\mathrm{Be}$ & $1 s^{2} 2 s^{2}$ & 28.892 & 29.364 & 29.368 \\
$\mathrm{~B}$ & $1 s^{2} 2 s^{2} p^{1}$ & 48.704 & 49.357 & 49.361 \\
$\mathrm{C}$ & $1 s^{2} 2 s^{2} p^{2}$ & 74.931 & 75.773 & 75.778 \\
$\mathrm{~N}$ & $1 s^{2} 2 s^{2} p^{3}$ & 108.258 & 109.291 & 109.297 \\
$\mathrm{O}$ & $1 s^{2} 2 s^{2} p^{4}$ & 149.042 & 150.284 & 150.289 \\
$\mathrm{~F}$ & $1 s^{2} 2 s^{2} p^{5}$ & 198.217 & 199.667 & 199.673 \\
$\mathrm{Ne}$ & $1 s^{2} 2 s^{2} p^{6}$ & 256.455 & 258.115 & 258.122 \\
$\mathrm{Na}$ & {$[\mathrm{Ne}] 3 s^{1}$} & 322.881 & 324.773 & 324.780 \\
$\mathrm{Mg}$ & {$[\mathrm{Ne}] 3 s^{2}$} & 398.265 & 400.399 & 400.407 \\
$\mathrm{Al}$ & {$[\mathrm{Ne}] 3 s^{2} p^{1}$} & 482.628 & 485.002 & 485.011 \\
$\mathrm{Si}$ & {$[\mathrm{Ne}] 3 s^{2} p^{2}$} & 576.429 & 579.047 & 579.056 \\
$\mathrm{P}$ & {$[\mathrm{Ne}] 3 s^{2} p^{3}$} & 679.991 & 682.855 & 682.865 \\
$\mathrm{~S}$ & {$[\mathrm{Ne}] 3 s^{2} p^{4}$} & 793.471 & 796.574 & 796.584 \\
$\mathrm{Cl}$ & {$[\mathrm{Ne}] 3 s^{2} p^{5}$} & 917.327 & 920.671 & 920.682 \\
$\mathrm{Ar}$ & {$[\mathrm{Ne}] 3 s^{2} p^{6}$} & 1051.876 & 1055.466 & 1055.477 \\
$\mathrm{~K}$ & {$[\mathrm{Ar}] 4 s^{1}$} & 1196.393 & 1200.244 & 1200.256 \\
$\mathrm{Ca}$ & {$[\mathrm{Ar}] 4 s^{2}$} & 1351.466 & 1355.583 & 1355.595 \\
\hline \hline
\end{tabular}


note that the LDA atomic total energies are always too small and the absolute error increases although the percentage error decreases as the atomic number increases. It is obvious from Fig. 1 that the GGA gives much better atomic total energies. It is interesting to compare the last two columns of Table II. The atomic energies calculated fully self-consistently within the GGA formulation are listed in the last column, whereas the other column

TABLE III. (a) First ionization energies in eV. Experimental data are from Ref. 35. (b) Removal energies of outlying shells of electrons (in $\mathrm{eV}$ ). The electron shells removed are indicated below. Experimental values are sum of ionization energies quoted in Ref. 35, except for $\mathrm{Ne}$, where the data are taken from Ref. 36.

\begin{tabular}{|c|c|c|c|}
\hline & & & \\
\hline Element & Expt. & LDA & GGA \\
\hline $\mathbf{H}$ & 13.60 & 13.03 & 13.67 \\
\hline $\mathrm{He}$ & 24.48 & 24.28 & 24.99 \\
\hline $\mathbf{L i}$ & 5.39 & 5.47 & 5.64 \\
\hline $\mathrm{Be}$ & 9.32 & 9.04 & 9.24 \\
\hline B & 8.30 & 8.56 & 8.66 \\
\hline $\mathrm{C}$ & 11.26 & 11.72 & 11.77 \\
\hline $\mathbf{N}$ & 14.53 & 14.94 & 14.94 \\
\hline $\mathrm{O}$ & 13.61 & 13.99 & 14.11 \\
\hline $\mathbf{F}$ & 17.42 & 18.11 & 18.12 \\
\hline $\mathrm{Ne}$ & 21.56 & 22.20 & 22.14 \\
\hline $\mathrm{Na}$ & 5.14 & 5.37 & 5.52 \\
\hline $\mathrm{Mg}$ & 7.64 & 7.74 & 7.93 \\
\hline Al & 5.98 & 5.99 & 6.06 \\
\hline $\mathrm{Si}$ & 8.15 & 8.25 & 8.32 \\
\hline $\mathbf{P}$ & 10.48 & 10.50 & 10.58 \\
\hline $\mathbf{S}$ & 10.36 & 10.62 & 10.52 \\
\hline $\mathrm{Cl}$ & 13.01 & 13.29 & 13.22 \\
\hline $\mathrm{Ar}$ & 15.76 & 15.95 & 15.93 \\
\hline K & 4.34 & 4.53 & 4.66 \\
\hline $\mathrm{Ca}$ & 6.11 & 6.23 & 6.39 \\
\hline & & & \\
\hline $\begin{array}{c}\text { Shell } \\
\text { removed }\end{array}$ & Expt. & LDA & GGA \\
\hline $1 s$ & 13.60 & 13.03 & 13.67 \\
\hline $1 s$ & 78.88 & 77.13 & 79.31 \\
\hline $2 s$ & 5.39 & 5.47 & 5.65 \\
\hline $2 s$ & 27.53 & 27.29 & 27.76 \\
\hline $2 s, 2 p$ & 71.37 & 71.03 & 71.81 \\
\hline $2 s, 2 p$ & 147.98 & 147.70 & 148.82 \\
\hline $2 s, 2 p$ & 266.86 & 266.57 & 268.02 \\
\hline $2 s, 2 p$ & 432.96 & 432.52 & 434.48 \\
\hline $2 s, 2 p$ & 658.65 & 658.23 & 660.69 \\
\hline $2 s, 2 p$ & 953.59 & 952.83 & 955.80 \\
\hline $3 s$ & 5.14 & 5.37 & 5.52 \\
\hline $3 s$ & 22.68 & 23.09 & 23.43 \\
\hline $3 s, 3 p$ & 53.25 & 53.72 & 54.15 \\
\hline $3 s, 3 p$ & 103.11 & 103.65 & 104.14 \\
\hline $3 s, 3 p$ & 176.72 & 177.36 & 177.90 \\
\hline $3 s, 3 p$ & 276.58 & 277.04 & 277.47 \\
\hline $3 s, 3 p$ & 408.98 & 408.96 & 409.28 \\
\hline $3 s, 3 p$ & 577.83 & 577.43 & 577.71 \\
\hline $4 s$ & 4.34 & 4.53 & 4.66 \\
\hline $4 s$ & 17.98 & 18.34 & 18.19 \\
\hline
\end{tabular}

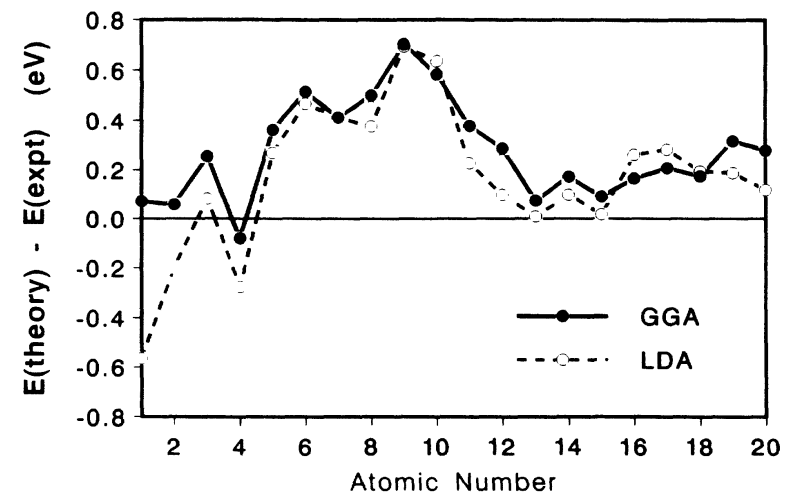

FIG. 2. Differences between theoretical and experimental [ $E$ (theory) $-E$ (expt)] first ionization energies calculated using the LDA (open circles) and the GGA (solid circles). Experimental data are from Ref. 35 . The lines serve as guides to the eye.

[GGA (NSC)] contains atomic energies computed by putting self-consistent LDA charge densities into the GGA energy functional for exchange and correlation. There differences are rather small. The calculated first ionization energies are listed in Table III(a) and their differences with experimental values are plotted in Fig. 2. The ionization energies are computed as the difference between the total energies of an atom and its positive ion (one electron less), both in their respective lowest-energy configurations. We note that the LDA first ionization energies are already in reasonable agreement with experimental values. Unlike the total energies, the GGA is not giving better first ionization energies than the LDA. We note that Perdew, Harbola, and Sahni ${ }^{22}$ have reached the same conclusion. We then calculate the electron removal energies of the outer valence-electron shells by stripping the atoms of electrons down to an inert-gas configuration. These electrons are those that govern the structural and cohesive properties of the elements in the crystalline state. Results are given in Table III(b), and are compared with experimental values in Fig. 3. Again we can see that

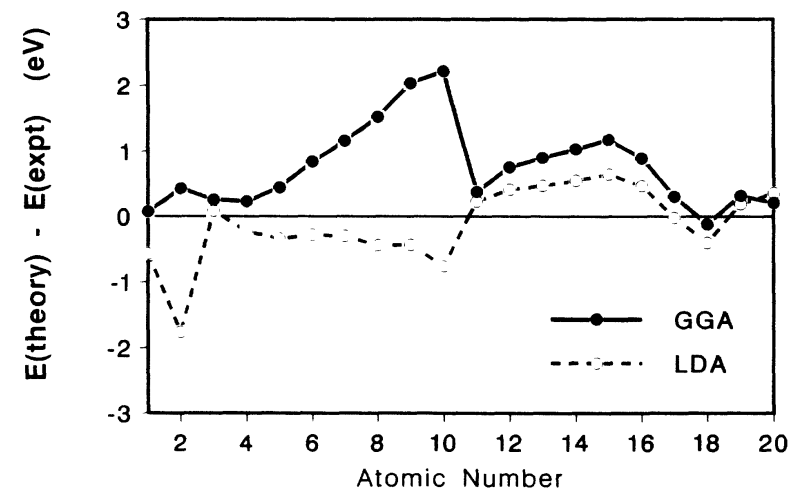

FIG. 3. Differences between theoretical and experimental [E(theory) $-E$ (expt)] removal energies for electrons in valence shells calculated using the LDA (open circles) and the GGA (solid circles). Experimental data are from Refs. 35 and 36. The lines serve as guides to the eye. 
the GGA does not seem to be superior to the LDA, and in fact, it gives slightly larger discrepancies for most elements calculated. Since the total energy of an atom is basically the energy needed to remove all its electrons, the fact that the GGA gives much better total energies while the LDA gives slightly better (at least not worse) removal
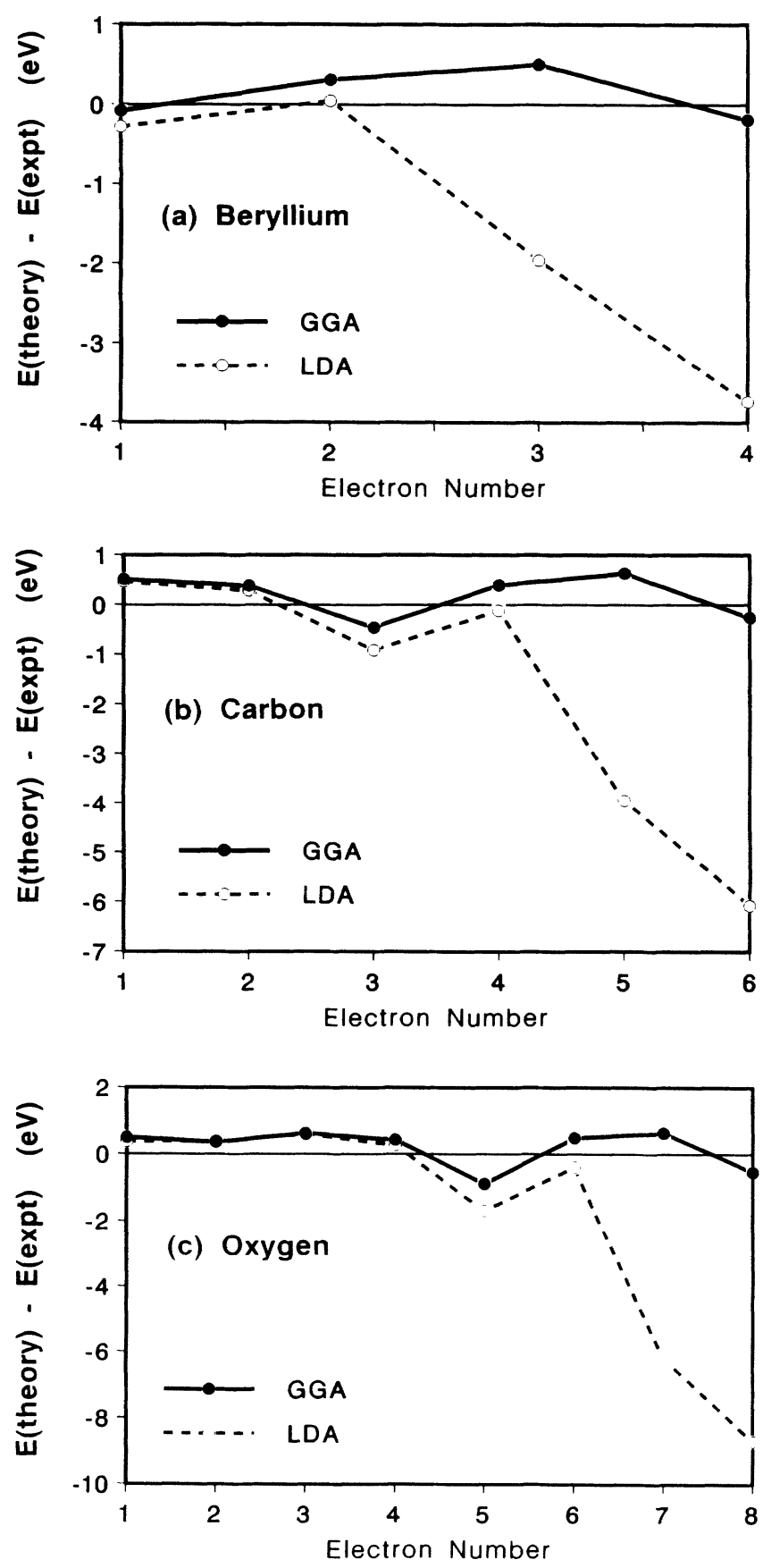

FIG. 4. Errors [ $E$ (theory) $-E$ (expt)] in calculated electron removal energies for (a) beryllium, (b) carbon, and (c) oxygen atoms. The removal energy for the $n$th electron is calculated as the difference between the energy of an ion with $n$ electrons and that with one electron less. The lines are guides for the eye only. Experimental data are from Ref. 35. Note that the GGA is much more accurate for core electrons. energies for the electrons in the outermost shells, implies that the GGA must be vastly superior for the core electrons. It is found to be indeed the case. We calculate the energy needed to remove an element of its electrons one by one down to the bare ion. Results are compared with experimental values in Fig. 4 for $\mathrm{Be}, \mathrm{C}$, and $\mathrm{O}$. We note that for the outermost electrons, the LDA and GGA results are more or less the same in the energy scale considered. On the other hand, the LDA seriously underestimates the binding energy of the core electrons, whereas the GGA does a much better job. Hence, we see that the LDA gives a much larger error (in an absolute scale) for the more tightly bound innermost core electrons, while the density-gradient corrections in the GGA compensate for most of the errors so that the error is of the same magnitude for core and valence electrons.

\section{SOLID CALCULATIONS}

We have calculated the structural and cohesive properties of $\mathrm{Al}, \mathrm{Si}$, and $\mathrm{C}$ using the GGA. These elements are chosen as prototypes for a few reasons. Firstly, this group of elements includes a simple metal, a semiconductor, and an insulator, each has rather distinct electronic properties. The valence-charge density varies from the "free-electron"-like character in Al to the more tightly bound character in $\mathrm{C}$ and the rather different valencecharge density gradients in these systems should offer a good test for the applicability of the GGA. Secondly, these elements have been studied carefully with the LDA before. Their calculated properties and the methods of calculation are well known and well established, so we can focus our attention on the comparison between the LDA and GGA results. Norm-conserving pseudopotentials $^{23}$ are used for all the calculations. Quantities like charge densities and total energies reported below, unless otherwise specified, are hence "pseudo"-quantities, i.e., quantities calculated using pseudopotentials. We use the pseudopotential-plane-wave $\operatorname{method}^{24}$ for $\mathrm{Al}$ and the pseudopotential local-orbital-method ${ }^{25,26}$ for $\mathrm{Si}$ and C. The former employs a plane-wave basis set to expand the electronic wave functions while the latter uses a basis set comprised of a Bloch sum of atomiclike orbitals. These basis sets are known to be very efficient for the elements under investigation. In both the plane-wave and the local-orbital approach, we express the charge density in the momentum space. This is advantageous for GGA calculations because the density gradients can be calculated rather straightforwardly in momentum space and transformed to real space using fast-Fourier-transform techniques. The total energies of the systems ( $\mathrm{Al}$ in the fcc structure, $\mathrm{Si}$ and $\mathrm{C}$ in the diamond structure) are calculated as a function of volume. The results are fitted to universal binding curves, ${ }^{27}$ which give the equilibrium lattice constants, bulk moduli, and total energies at equilibrium. The cohesive energies are then found by subtracting off the zero-point energies of the solids and the reference energies of the isolated spin-polarized atoms in their ground-state configurations. We note that the spinpolarization energies are always deduced from allelectron calculations. The zero-point energy of $\mathrm{Al}$ is es- 
TABLE IV. Cohesive and structural properties of Al calculated using the LDA (Ceperley-Alder) and the GGA. The GGA (NSC) is calculated by putting LDA charge densities into the GGA exchange-correlation function. A zero-point energy of $0.042 \mathrm{eV}$ is included in the calculation of cohesive energy. Experimental values are taken from Ref. 37.

\begin{tabular}{lcccc}
\hline \hline & Expt. & LDA & GGA & GGA (NSC) \\
\hline Cohesive energy (eV) & 3.39 & 4.01 & 3.38 & 3.36 \\
Lattice constant (̊) & 4.05 & 3.96 & 3.94 & 3.95 \\
Bulk modulus (Mbar) & 0.72 & 0.84 & 0.87 & 0.88 \\
\hline \hline
\end{tabular}

timated within a Debye model ${ }^{28}$ to be $0.042 \mathrm{eV}$ per atom. For $\mathrm{C}$ and $\mathrm{Si}$, the zero-point energies are found to be 0.195 and $0.065 \mathrm{eV}$, respectively, and are calculated from theoretical phonon spectra deduced from a tight-binding force model, ${ }^{29}$ which gives very good structural and lattice vibrational properties for $\mathrm{Si}$ and $\mathrm{C}$.

For Al, the LDA calculations using the pseudopotential-plane-wave method have given excellent results for structural properties, phonon frequencies, surface relaxations, and surface vibrational properties. ${ }^{30}$ Cohesive energy overbinds by over $0.5 \mathrm{eV}$ within the LDA. We will see how the GGA affects the structural and cohesive properties. Following previous calculations, we use the pseudopotential-plane-wave approach. The electronic wave functions are expanded in a plane-wave basis with kinetic energy up to $8.5 \mathrm{Ry}$, and plane waves up to $16 \mathrm{RY}$ are included via Löwdin perturbation. ${ }^{31}$ We used $60 \mathrm{k}$ points in the irreducible wedge of the Brillouin zone to determine the charge density and a Gaussian weighting scheme $^{32}$ is used to determine the occupancy of the electron states near the Fermi level. Results for the cohesive energy, lattice constant, and bulk modulus of $\mathrm{Al}$ in the fcc structure calculated self-consistently with the LDA (Ceperley-Alder) are shown in the second column in Table IV. Our tests show that the LDA results are actually slightly better when computed with the Wigner ${ }^{4}$ form of correlation but since the GGA improves upon the Ceperley-Alder form, we show the LDA results computed with the Ceperley-Alder form for a more meaningful comparison with the GGA results. Results calculated self-consistently with the GGA are shown in the third column. We see that the changes in lattice constant and bulk modulus are very small, preserving the good agreement with experimental results. The cohesive energy calculated with GGA is in excellent agreement with experiment, correcting for the overbinding of the LDA cohesive energy. We found that most of the change in the cohesive energy originates from the change in the energy of the isolated atom. The (pseudo)atom energies cal- culated with the GGA are lower than those calculated using the LDA by $0.684 \mathrm{eV}$, while the GGA only lowers the energy of the solid by $0.053 \mathrm{eV}$. In fact, the GGA changes the energy of the solid by such a small amount that the changes in structural properties are negligible. This is understandable because the valence electrons in the Al crystal, more or less free-electron-like, have very smooth pseudocharge density and hence the gradient correction in the exchange and correlation energy is very small. Results calculated by putting the self-consistent LDA charge densities into the GGA functionals for the evaluation of exchange and correlation energies are listed in the last column of Table IV. They are very close to those obtained self-consistently within the GGA formulation. This means that the GGA correction does not change the charge density to a large extent. In fact, when we examine the charge density contour plots, we find that the self-consistent GGA and LDA charge densities are almost visually indistinguishable. The variational property of the total energy also guarantees that the error in the charge density only contributes to error in the total energy in the second order. Hence, self-consistency in the GGA formulation seems relatively unimportant in this case.

For $\mathrm{C}$ and $\mathrm{Si}$, we use a local-orbital pseudopotential method. ${ }^{25,26}$ The method has been described in detail previously and has been rather successful with semiconductors, insulators, and transition-metal systems. The basis consisted of Bloch sums of atomiclike orbitals, which has the form

$$
\phi_{\mu \alpha l m}(\mathbf{k}, \mathbf{r})=\frac{1}{\sqrt{\Omega}} \sum_{\mathbf{R}} e^{i \mathbf{k} \cdot\left(\mathbf{R}+\mathbf{r}_{\mu}\right)} f_{\alpha l m}\left(\mathbf{r}-\mathbf{R}-\tau_{\mu}\right)
$$

where $\Omega$ is the volume of the crystal, $\mathbf{R}$ is a lattice vector, $\tau_{\mu}$ is a basis vector, and $f(r)$ are atomiclike functions of the following form: $f(r)=A e^{-\alpha r^{2}} r^{l} K_{l m}(\theta, \psi)$, where $A$ is a normalization constant. The coefficients $\alpha$ in the Gaussian radial functions are chosen to minimize the to-

TABLE V. Cohesive and structural properties of $\mathrm{C}$ calculated using the LDA (Ceperley-Alder) and the GGA. The GGA (NSC) is calculated by putting LDA charge densities into the GGA exchangecorrelation functional. A zero-point energy of $0.195 \mathrm{eV}$ is included in the calculation of cohesive energy. Experimental values are taken from Ref. 37.

\begin{tabular}{lllll}
\hline \hline & Expt. & CA & GGA & GGA (NSC) \\
\hline Cohesive energy $(\mathrm{eV})$ & 7.37 & 8.21 & 7.35 & 7.32 \\
Lattice constant $(\AA)$ & 3.567 & 3.55 & 3.58 & 3.59 \\
Bulk modulus $(\mathrm{Mbar})$ & 4.42 & 4.49 & 4.13 & 4.11 \\
\hline \hline
\end{tabular}


TABLE VI. Cohesive and structural properties of Si calculated using the LDA (Ceperley-Alder) and the GGA. The GGA (NSC) is calculated by putting LDA charge densities into the GGA exchangecorrelation functional. A zero-point energy of $0.065 \mathrm{eV}$ is included in the calculation of cohesive energy. Experimental values are taken from Ref. 37.

\begin{tabular}{lcccc}
\hline & Expt. & CA & GGA & GGA (NSC) \\
\hline Cohesive energy (eV) & 4.63 & 5.33 & 4.55 & 4.53 \\
Lattice constant (̊) & 5.43 & 5.35 & 5.39 & 5.39 \\
Bulk modulus (Mbar) & 0.99 & 1.19 & 1.10 & 1.09 \\
\hline \hline
\end{tabular}

tal energy of the system under consideration. We use the set of coefficients $\alpha=\{1.75,0.85,0.41,0.20\}$ (with $r$ in atomic units) for $\mathrm{Si}$ and the set $\{3.10,1.22,0.48,0.19\}$ for C. We include cubic harmonics up to $l=1(s, p)$ for $\mathbf{C}$ and $l=2(s, p, d)$ for Si. Although it is difficult to gauge the absolute convergence of a linear-combination-ofatomic-orbitals-type basis, tests based on varying the number and values of the Gaussian coefficients indicate that we are converged to about $0.1 \mathrm{eV}$ for $\mathrm{Si}$ and $0.2 \mathrm{eV}$ for $C$. Ten special $\mathbf{k}$ points ${ }^{33}$ in the irreducible part of the Brillouin zone are used for computing charge densities and the sum of eigenvalues. Cohesive energy, lattice constant, and bulk modulus are calculated for $\mathrm{C}$ and $\mathrm{Si}$ with both the LDA (with the Ceperley-Alder form of correlation) and the GGA. Results are shown in Tables $V$ and VI. Again, we see that the LDA (Ref. 34) gives good lattice constants and bulk moduli and overbinds the cohesive energies. The GGA results change the structural properties only by a small amount but substantially improve the cohesive energies for both $\mathrm{Si}$ and $\mathrm{C}$. Just as in the case of Al, the GGA correction gives lower energy for both the isolated atom and solid, but lowers the energy in the atom more than the solid, compensating for the overbinding. For C, the GGA lowers the energy in the isolated (pseudo)atom by $1.326 \mathrm{eV}$ and the solid by 0.465 $\mathrm{eV}$, while for $\mathrm{Si}$, the atomic energy is lowered by $1.221 \mathrm{eV}$ and the solid by $0.446 \mathrm{eV}$. The valence charge densities in $\mathrm{Si}$ and $\mathrm{C}$ have directional bondings, and are not as smooth as the charge densities in Al. This contributes to larger gradient corrections in the energy of the crystal than that found in Al. Results calculated by putting LDA charge densities into the GGA functional are again very close to those calculated self-consistently within the framework of the GGA. This can be seen by comparing the last two columns in Tables $\mathrm{V}$ and VI.

\section{DISCUSSION}

The main purpose of this paper is to apply the GGA to study the cohesive and structural properties of $\mathrm{Al}, \mathrm{Si}$, and C. For all three of them, the GGA corrects for the overbinding of cohesive energies in the LDA, giving excellent cohesive energies when compared with experiments. The changes in the structural properties such as the equilibrium lattice constants are relatively small. GGA has been applied to improve the total energy of atoms and dissociation energy of molecules, ${ }^{17}$ and here we show that it is also good for the cohesive energy for solids.

The question then is why the GGA should work so well in describing the cohesive energies, at least for the elements we have tested. It does not seem to be a coincidence, as the three elements we considered have rather different electronic properties, and yet the GGA gives very good cohesive energies for all three. We know from numerous previous calculations that the LDA almost always overestimates cohesive energies. The exchangecorrelation contributions due to the gradient terms of the GGA lower the total energy of the system with respect to the energy given by the LDA. This can be seen directly from the GGA expressions of exchange and correlation, noting that the exchange term is usually dominant, and is also evident from Table II. Total energies computed with GGA will thus be lower than those given by the LDA for both the atom and the solid, but we expect the GGA to lower the energy of the atom more than the energy of the solid. This is because the charge-density gradient is expected to be smaller in a bulk-crystalline environment than in the isolated atomic environment, as the valence electrons smear out through the solid. As the energy of the atom is lowered with respect to the solid, the cohesive energy deduced from the GGA will be smaller than that from the LDA, thus correcting the overbinding in the right direction. This is basically what we have observed for $\mathrm{Al}, \mathrm{Si}$, and $\mathrm{C}$. However, we should not have the illusion that the GGA is correct to the order of $0.1 \mathrm{eV}$ on an absolute scale, either for atomic or crystalline environments. There is no doubt that the GGA gives better atomic total energies than the LDA, but the absolute error (as compared with the exact atomic total energy) is still nontrivial. If we focus on the description of the valence electrons, we can see from Table III(b) or Fig. 3 that the GGA is making an error of the order of $1 \mathrm{eV}$ for the elements under consideration. Thus, the good cohesive energy obtained is still mainly a consequence of error cancellation properties, rather than the ability to describe the absolute value of the total energy. If we take a second look at Figs. 4(a)-4(c), we can see that the LDA gives very reasonable binding energies for valence electrons but substantially underestimates the binding of tightly bound core electrons. On the other hand, the GGA is about equally good for valence and core electrons. This may serve as evidence that the GGA is making more or less the same error for the binding energy of electrons under a different environment and it is then not surprising that most of these errors cancel out if we are interested in relative energies (e.g., cohesive energy) rather than absolute energies.

All cohesive energies in this paper are computed with pseudopotentials. Previous experience has suggested that modern norm-conserving pseudopotentials ${ }^{23}$ give 
cohesive properties very close to all-electron results, if both calculations are equally well converged. It is, however, rather difficult to give $a$ priori error estimates for pseudopotentials. As long as the valence electrons are well separated from the core electrons, we may view pseudopotential as an implementation of the "frozencore" approximation. For $\mathrm{Si}$, the calculation of Harmon, Weber and Hamann ${ }^{38}$ show that frozen core results of the cohesive energy differs from all-electron results by less than $0.1 \mathrm{eV}$. It is thus reasonable to believe that the conclusion reached in this paper about cohesive energies will not change if the results are computed with all-electron calculations.

If the eigenvalues from solving the Kohn-Sham equations within the LDA are interpreted as quasiparticle energies, the energy gaps for semiconductors and insulators are consistently underestimated by as much as $30-50 \%$. When we compare the band structures for $\mathrm{Si}$ and $\mathrm{C}$ obtained using the GGA with those obtained with the LDA, we find that the GGA gives larger eigenvalue differences and, hence, larger gaps. For instance, the indirect gap of $\mathrm{Si}$ is increased by $0.32 \mathrm{eV}$ while the direct gap is increased by $0.14 \mathrm{eV}$. The GGA thus improves the gaps when compared with experimental values. However, we do not want to put too much emphasis on this aspect since even the "exact" density-functional eigenvalues are not supposed to give the correct fundamental gaps. The proper way to obtain quasiparticle energies is to compute the self-energy operator. ${ }^{39}$

Lastly, we note that the atomic reference energies used in the calculation of cohesive energies are calculated from "spherical" atoms. In LDA calculations, one seldom worries about nonspherical corrections in the atomic total energy since the correction is usually much smaller than the error in the calculated cohesive energy and the correction is not systematic (the correction can either be positive or negative). Kutzler and Painter $^{17}$ calculated atomic energies for B, C, O, and F and found that the gradient corrections give systematically lower energies for the nonspherical atoms. For $\mathrm{C}$, the removal of the spherical approximation lowers the energy by $0.113 \mathrm{eV}$. So, if one worries about cohesive energies down to the $0.1-\mathrm{eV}$ level, the nonspherical corrections should be taken into account.

There are still many areas that the applicability of the GGA need to be tested. Some examples are cohesive properties of transition-metal elements, magnetic properties, and phonon frequencies computed using the frozenphonon method. For the moment, the results look encouraging. It seems that the GGA offers a rather painless way of improving the systematic overbinding problem of LDA calculations.

\section{ACKNOWLEDGMENTS}

We are grateful to Dr. G. S. Painter for kindly sending us some unpublished information. We thank C. Elsaesser for communicating Ref. 20 and for his help in testing some programs. We thank Ms. C. H. Xu for calculating the zero-point energies of Si and C. Ames Laboratory is operated for the U.S. Department of Energy by Iowa State University under Contract No. W-7405-ENG-82. This work is supported by the Director of Energy Research, Office of Basic Energy Sciences.
*Permanent address: Institute of Theoretical Physics, Hebei Normal University, Shijiazhuang, Hebei, People's Republic of China.

†Permanent address: Department of Physics, Wuhan University, Wuhan, Hubei, People's Republic of China.

${ }^{1}$ P. Hohenberg and W. Kohn, Phys. Rev. 136, B864 (1964).

${ }^{2}$ W. Kohn and L. J. Sham, Phys. Rev. 140, A1133 (1965).

${ }^{3}$ For a review, see, e.g., Theory of the Inhomogeneous Electron Gas, edited by N. H. March and S. Lundqvist (Plenum, New York, 1983).

${ }^{4}$ E. Wigner, Phys. Rev. 46, 1002 (1934).

${ }^{5}$ L. Hedin and B. I. Lundqvist, J. Phys. C4, 2064 (1971).

${ }^{6}$ D. M. Ceperley and B. J. Alder, Phys. Rev. Lett. 45, 566 (1980).

${ }^{7}$ P. Perdew and A. Zunger, Phys. Rev. B 23, 5048 (1981).

${ }^{8}$ For a recent review, see, e.g., R. O. Jones and O. Gunnarsson, Rev. Mod. Phys. 61, 689 (1989).

${ }^{9}$ S.-k. Ma and K. A. Brueckner, Phys. Rev. 165, 18 (1968).

${ }^{10}$ O. Gunnarsson and B. I. Lundqvist, Phys. Rev. B 13, 4274 (1976).

${ }^{11}$ D. C. Langreth and M. J. Mehl, Phys. Rev. Lett. 47, 446 (1981); Phys. Rev. B 28, 1809 (1983); 29, 2310(E) (1984).

${ }^{12}$ C. D. Hu and D. C. Langreth, Phys. Scr. 32, 391 (1985).

${ }^{13}$ J. P. Perdew, Phys. Rev. Lett. 55, 1665 (1985).

14J. P. Perdew and W. Yue, Phys. Rev. B 33, 8800 (1986).
15J. P. Perdew, Phys. Rev. B 33, 8822 (1986); 34, 7406(E) (1986).

${ }^{16}$ C. D. Hu and D. C. Langreth, Phys. Rev. B 33, 943 (1986).

${ }^{17}$ F. W. Kutzler and G. S. Painter, Phys. Rev. Lett. 59, 1285 (1987); Phys. Rev. B 37, 2850 (1988).

${ }^{18} \mathrm{U}$. von Barth and R. Car, in The Electronic Structure of Complex Systems, Vol. 113 of NATO Advanced Studies Institute Series B: Physics, edited by P. Phariseau and W. M. Temmerman (Plenum, New York, 1984), p. 67.

${ }^{19}$ U. von Barth and A. C. Pedroza, Phys. Scr. 32, 353 (1985).

${ }^{20}$ P. Bango, O. Jepsen, and O. Gunnarsson (unpublished).

${ }^{21}$ F. Herman and S. Skillman, Atomic Structure Calculations (Prentice-Hall, Englewood Cliffs, NJ, 1963).

22J. P. Perdew, M. K. Harbola, and V. Sahni, in Condensed Matter Theories, edited by J. S. Arponen, R. F. Bishop, and M. Manninen (Plenum, New York, 1988), Vol. 3, p. 235.

${ }^{23}$ D. H. Hamann, M. Schlüter, and C. Chiang, Phys. Rev. Lett. 43, 1494 (1979).

${ }^{24}$ J. Ihm, A. Zunger, and M. L. Cohen, J. Phys. C 12, 4409 (1979).

25J. R. Chelikowsky and S. G. Louie, Phys. Rev. B 29, 3470 (1984); J. R. Chelikowsky and S. G. Louie, D. Vanderbilt, and C. T. Chan, Int. J. Quantum Chem. Symp. 105, 105 (1984).

${ }^{26}$ C. T. Chan, D. Vanderbilt, and S. G. Louie, Phys. Rev. B 33, 2455 (1986); 34, 8791(E) (1986).

${ }^{27}$ J. H. Rose, J. Ferrante, and J. R. Smith, Phys. Rev. Lett. 47, 
675 (1981).

${ }^{28}$ V. L. Moruzzi, J. F. Janak, and A. R. Williams, Calculated Properties of Metals (Pergamon, New York, 1989).

${ }^{29}$ C. Z. Wang, C. T. Chan, and K. M. Ho, Phys. Rev. B 39, 8586 (1989); 40, 3390 (1989).

${ }^{30}$ P. K. Lam and M. L. Cohen, Phys. Rev. B 24, 4224 (1981); 25, 6139 (1982); K. M. Ho and K. P. Bohnen, Phys. Rev. Lett. 56, 934 (1986).

${ }^{31}$ P. Lowdin, J. Chem. Phys. 19, 1396 (1951).

${ }^{32}$ C. L. Fu and K. M. Ho, Phys. Rev. B 28, 5480 (1983).

${ }^{33}$ D. J. Chadi and M. L. Cohen, Phys. Rev. B 8, 5747 (1973).

${ }^{34}$ LDA results presented here are slightly different from those published before in Refs. 25 and 26 . We have used the Hedin-Lundqvist form of local exchange correlation previ- ously and the Ceperley-Alder form is used now. Also, for C, the basis set is more complete and the results presented here are fully self-consistent.

${ }^{35}$ CRC Handbook of Chemistry and Physics, 58th ed., edited by R. C. Weast (Chemical Rubber Co., Cleveland, 1977).

${ }^{36}$ C. E. Moore, Ionization Potential and Ionization Limits from The Analysis of Optical Spectra, U.S. Natl. Bur. Stand., Nat. Ref. Data Ser. No. 34 (U.S. GPO, Washington, D.C., 1970).

${ }^{37}$ C. Kittel, Introduction to Solid State Physics (Wiley, New York, 1976).

${ }^{38}$ B. N. Harmon, W. Weber, and D. H. Hamann, Phys. Rev. B 25, 1109 (1982).

${ }^{39}$ See, e.g., M. S. Hybertsen and S. G. Louie, Phys. Rev. Lett. 55, 1418 (1985). 